\title{
Antiperiodic Solutions to Impulsive Cohen-Grossberg Neural Networks with Delays on Time Scales
}

\author{
Yanqin Wang ${ }^{1,2}$ and Maoan Han ${ }^{1}$ \\ ${ }^{1}$ Applied Mathematics Department, Shanghai Normal University, Shanghai 200234, China \\ ${ }^{2}$ School of Mathematics and Physics, Changzhou University, Changzhou, Jiangsu 213164, China \\ Correspondence should be addressed to Maoan Han; mahan@shnu.edu.cn
}

Received 2 March 2014; Accepted 22 June 2014; Published 9 July 2014

Academic Editor: Qi-Ru Wang

Copyright (C) 2014 Y. Wang and M. Han. This is an open access article distributed under the Creative Commons Attribution License, which permits unrestricted use, distribution, and reproduction in any medium, provided the original work is properly cited.

\begin{abstract}
We use the method of coincidence degree and construct suitable Lyapunov functional to investigate the existence and global exponential stability of antiperiodic solutions of impulsive Cohen-Grossberg neural networks with delays on time scales. Our results are new even if the time scale $\mathbb{T}=\mathbb{R}$ or $\mathbb{Z}$. An example is given to illustrate our feasible results.
\end{abstract}

\section{Introduction}

It is well known that Cohen-Grossberg neural networks (CGNNs) include many models from different research fields, such as neurobiology, population biology, and evolutionary theory, as well as Hopfield neural networks and other recurrent neural network models. Over the past few years, a large number of scholars have extensively studied the dynamical behaviors, in particular, the existence and stability of the equilibrium point and periodic and almost-periodic solutions of Cohen-Grossberg neural networks. There have been considerable results on CGNNs (e.g., see [1-16]). In contrast, however, very few results are available on the existence and exponential stability of antiperiodic solutions for neural networks, while the existence of antiperiodic solutions plays a key role in characterizing the behavior of nonlinear differential equations (see [17-22]).

In [17], the authors studied the existence and exponential stability of antiperiodic solutions for the following CohenGrossberg neural networks with bounded and unbounded delays:

$$
\begin{aligned}
& \dot{x}_{i}(t) \\
& =-a_{i}\left(x_{i}(t)\right)\left[b_{i}\left(x_{i}(t)\right)-\sum_{j=1}^{n} c_{i j}(t) f_{j}\left(x_{j}(t)\right)\right.
\end{aligned}
$$

$$
\begin{aligned}
& -\sum_{j=1}^{n} d_{i j}(t) g_{j}\left(x_{j}\left(t-\tau_{i j}(t)\right)\right) \\
& -\sum_{j=1}^{n} e_{i j}(t) \int_{0}^{+\infty} K_{i j}(s) h_{j}\left(x_{j}(t-s)\right) \mathrm{d} s, \\
& \left.-I_{i}(t)\right], \quad t \neq t_{k}, t \geq 0,
\end{aligned}
$$

where $n$ corresponds to the number of units in the neural networks, $x_{i}$ denotes the potential (or voltage) of cell $i$ at time $t, a_{i}$ represents an amplification function, $b_{i}$ is an appropriately behaved function, $c_{i j}, d_{i j}$, and $e_{i j}$ denote the strengths of connectivity between cells $i$ and $j$ at time $t$, respectively. The activation functions $f_{j}, g_{j}$, and $h_{j}$ show how the $j$ th neuron reacts to the input, $\tau_{i j}$ corresponds to the time delay required in processing and transmitting a signal from the $j$ th cell to the $i$ th cell at time $t, K_{i j}$ is the kernel, and $I_{i}$ denotes the $i$ th component of an external input source introduced from outside the network to cell $i$ at time $t, i, j=$ $1,2, \ldots, n$. 
In fact, both continuous and discrete systems are very important in implementation and application. Therefore, the study of dynamic equations on time scales has received much attention (see [18, 19, 23-28]) which displays a combination of characteristics of both continuous-time and discrete-time system. For example, in [23], the authors extended the almost-periodic theory on time scales with the delta derivative to that with the nabla derivative and then derived some sufficient conditions ensuring the existence, uniqueness, and exponential stability of almost-periodic solutions for a class of cellular neural networks with time-varying delays in leakage terms on time scales.

Also, differential equations with impulses provide an adequate mathematical model of many evolutionary processes that suddenly change their states at certain moments. For example, [18] applied the method of coincidence degree to investigate the existence of antiperiodic solutions to the following impulsive shunting inhibitory cellular neural networks on time scales:

$$
\begin{aligned}
& x_{i j}^{\Delta}(t) \\
& =-a_{i j}(t) x_{i j}(t) \\
& \quad-\sum_{c_{k l} \in N_{r}(i, j)} c_{i j}^{k l}(t) \int_{0}^{+\infty} K_{i j}(u) x_{i j}(t) f_{i j}\left(x_{k l}(t-u)\right) \Delta u \\
& +L_{i j}(t), \quad t \in \mathbb{T}^{+}, t \neq t_{h}, h \in \mathbb{N}, \\
& \quad \Delta x_{i j}\left(t_{h}\right)=x_{i j}\left(t_{h}^{+}\right)-x_{i j}\left(t_{h}^{-}\right)=I_{i j k}\left(x_{i j}\left(t_{h}\right)\right), \\
& \quad t=t_{h}, \quad i=1,2, \ldots, m, \quad j=1, \ldots, n .
\end{aligned}
$$

Motivated by the abovementioned works, in this paper, we will apply the method of coincidence degree and construct suitable Lyapunov functional to investigate the existence and global exponential stability of antiperiodic solutions to the following impulsive CGNN model with delays on time scales:

$$
\begin{gathered}
x_{i}^{\Delta}(t) \\
=-\alpha_{i}\left(x_{i}(t)\right)\left[\beta_{i}\left(x_{i}(t)\right)-\sum_{j=1}^{n} a_{i j}(t) f_{j}\left(x_{j}(t)\right)\right. \\
-\sum_{j=1}^{n} b_{i j}(t) g_{j}\left(x_{j}\left(t-\tau_{i j}(t)\right)\right) \\
-\sum_{j=1}^{n} c_{i j}(t) \int_{0}^{+\infty} K_{i j}(s) h_{j}\left(x_{j}(t-s)\right) \Delta s \\
\left.+J_{i}(t)\right], \quad t \in \mathbb{T}^{+}, t \neq t_{k}, k \in \mathbb{N}, \\
\Delta x_{i}\left(t_{k}\right)=x_{i}\left(t_{k}^{+}\right)-x_{i}\left(t_{k}^{-}\right)=I_{i k}\left(x_{i}\left(t_{k}\right)\right), \\
t=t_{k}, \quad i=1,2, \ldots, n,
\end{gathered}
$$

where $\mathbb{T}$ is an $(\omega / 2)$-periodic time scale which has the subspace topology inherited from the standard topology on $\mathbb{R}, \mathbb{T}^{+}=\{t \in \mathbb{T}: t \geq 0\}, x_{i}\left(t_{k}^{+}\right), x_{i}\left(t_{k}^{-}\right)$represent right and left limit of $x_{i}\left(t_{k}\right)$ in the sense of time scales, $t_{k}$ is a sequence of real numbers such that $0<t_{1}<t_{2}<\cdots<t_{k} \rightarrow \infty$ as $k \rightarrow \infty$, and there exists a positive integer $\mathrm{p}$ such that $t_{k+p}=$ $t_{k}+\omega / 2, I_{i(k+p)}\left(x_{i}\left(t_{k+p}\right)\right)=-I_{i k}\left(-x_{i}\left(t_{k}\right)\right), k \in \mathbb{N}$. Without loss of generality, we also assume that $[0, \omega / 2)_{\mathbb{T}} \bigcap\left\{t_{k}: k \in \mathbb{N}\right\}=$ $\left\{t_{1}, t_{2}, \ldots, t_{q}\right\}$. form

The initial conditions associated with system (3) are of the

$$
x_{i}(s)=\varphi_{i}(s), \quad s \in(-\infty, 0]_{\mathbb{T}}, i=1,2, \ldots, n,
$$

where $\varphi_{i}(s)$ is a bounded continuous function on $(-\infty, 0]_{\mathbb{T}}$.

Throughout this paper, we make the following assumptions:

$\left(\mathrm{H}_{1}\right) J_{i}, a_{i j}, b_{i j}, c_{i j}$, and $\tau_{i j} \in C(\mathbb{T}, \mathbb{R}), a_{i j}(t+\omega / 2)=$ $a_{i j}(t), b_{i j}(t+\omega / 2)=b_{i j}(t), c_{i j}(t+\omega / 2)=c_{i j}(t)$, $\tau_{i j}(t+\omega / 2)=\tau_{i j}(t), J_{i}(t+\omega / 2)=-J_{i}(t)$, and $\left|J_{i}(t)\right|<$ $\infty, i, j=1,2, \ldots, n$;

$\left(\mathrm{H}_{2}\right) \quad \alpha_{i} \in C\left(\mathbb{R}, \mathbb{R}^{+}\right), \alpha_{i}(-u)=\alpha_{i}(u)$, and there exist positive constants $\alpha_{i}^{m}, \alpha_{i}^{M}, \alpha_{i}^{L}$ such that $\alpha_{i}^{m} \leq \alpha_{i}(u) \leq$ $\alpha_{i}^{M}$ and $\left|\alpha_{i}\left(v_{1}\right)-\alpha_{i}\left(v_{2}\right)\right| \leq \alpha_{i}^{L}\left|v_{1}-v_{2}\right|$ for all $u, v_{1}, v_{2} \in$ $\mathbb{R}, i=1,2, \ldots, n$;

$\left(\mathrm{H}_{3}\right) \quad \beta_{i} \in C(\mathbb{R}, \mathbb{R})$ is delta differential and $0<\rho_{i} \leq \beta_{i}^{\Delta} \leq$ $\delta_{i}, \beta_{i}(-u)=-\beta_{i}(u), \beta_{i}(0)=0, \quad i=1,2, \ldots, n ;$

$\left(\mathrm{H}_{4}\right)$ there exists a positive constant $\alpha_{i}^{l}$ such that $\left|\alpha_{i}(u) \beta_{i}(u)-\alpha_{i}(v) \beta_{i}(v)\right|>\alpha_{i}^{l}\left|\beta_{i}(u)-\beta_{i}(v)\right|$ for all $u, v \in \mathbb{R}$

$\left(\mathrm{H}_{5}\right) f_{j}, g_{j}$, and $h_{j} \in C(\mathbb{R}, \mathbb{R}), f_{j}(-u)=-f_{j}(u), g_{j}(-u)=$ $-g_{j}(u), h_{j}(-u)=-h_{j}(u), f_{j}(0)=g_{j}(0)=h_{j}(0)=0$, and there exist positive constants $f_{j}^{M}, g_{j}^{M}, h_{j}^{M}, f_{j}^{L}, g_{j}^{L}$, and $h_{j}^{L}$ such that $\left|f_{j}(u)\right| \leq f_{j}^{M},\left|g_{j}(u)\right| \leq g_{j}^{M},\left|h_{j}(u)\right| \leq$ $h_{j}^{M},\left|f_{j}\left(v_{1}\right)-f_{j}\left(v_{2}\right)\right| \leq f_{j}^{L}\left|v_{1}-v_{2}\right|,\left|g_{j}\left(v_{1}\right)-g_{j}\left(v_{2}\right)\right| \leq$ $g_{j}^{L}\left|v_{1}-v_{2}\right|$, and $\left|h_{j}\left(v_{1}\right)-h_{j}\left(v_{2}\right)\right| \leq h_{j}^{L}\left|v_{1}-v_{2}\right|$, for all $u, v_{1}, v_{2} \in \mathbb{R}, j=1,2, \ldots, n$;

$\left(\mathrm{H}_{6}\right) \int_{0}^{+\infty}\left|K_{i j}(u)\right| \Delta u<+\infty, i, j=1,2, \ldots, n$

$\left(\mathrm{H}_{7}\right) \quad I_{i k} \in C(\mathbb{R}, \mathbb{R})$ and there exist positive constants $\rho_{i k}$ such that $\left|I_{i k}(u)-I_{i k}(v)\right| \leq \rho_{i k}|u-v|$ for all $u, v \in \mathbb{R}$, $k \in \mathbb{N}$, and $i=1,2, \ldots, n$.

For the sake of convenience, we introduce some notations:

$$
\begin{array}{ll}
a_{i j}^{M}=\max _{t \in[0, \omega]_{\mathbb{T}}} a_{i j}(t), & a_{i j}^{m}=\min _{t \in[0, \omega]_{\mathbb{T}}} a_{i j}(t), \\
J_{i}^{M}=\max _{t \in[0, \omega]_{\mathbb{T}}} J_{i}(t), & \tau=\sup _{t \in[0, \omega]_{\mathbb{T}}} \max _{1 \leq i, j \leq n}\left\{\tau_{i j}(t)\right\},
\end{array}
$$




$$
\begin{gathered}
B_{i}=\sum_{j=1}^{n} a_{i j}^{M} f_{j}^{M}+\sum_{j=1}^{n} b_{i j}^{M} g_{j}^{M}+\sum_{j=1}^{n} c_{i j}^{M} h_{j}^{M} \int_{0}^{\infty}\left|K_{i j}(s)\right| \Delta s, \\
E_{i}=\rho_{i} \alpha_{i}^{m} \omega\left(1-\alpha_{i}^{M} \delta_{i} \omega\right)-\left(1+\rho_{i} \alpha_{i}^{m} \omega\right) \sum_{k=1}^{2 q} \rho_{i k}, \\
D_{i}=\left(1+\alpha_{i}^{m} \rho_{i} \omega\right)\left(\alpha_{i}^{M} \omega\left(B_{i}+J_{i}^{M}\right)+\sum_{k=1}^{2 q}\left|I_{i k}(0)\right|\right), \\
R_{i}=\sum_{j=1}^{n}\left(a_{i j}^{M} f_{j}^{L}+b_{i j}^{M} g_{j}^{L} e_{\varepsilon}\left(t, t-\tau_{i j}(t)\right)\right. \\
\left.+c_{i j}^{M} h_{j}^{L} \int_{0}^{+\infty}\left|K_{i j}(s)\right| e_{\varepsilon}(t, t-s) \Delta s\right),
\end{gathered}
$$

where $i, j=1,2, \ldots, n$.

The organization of the rest of this paper is as follows. In Section 2, we introduce some definitions and make some preparations for later sections. In Sections 3 and 4, we establish our main results for the existence and exponential stability of antiperiodic solutions of (3). Finally, we present an example to illustrate the feasibility and effectiveness of our results obtained in previous sections.

\section{Preliminaries}

In this section, we recall some basic definitions and lemmas which are used in what follows.

Definition 1 (see $[24,26]$ ). A time scale $\mathbb{T}$ is an arbitrary nonempty closed subset of the real set $\mathbb{R}$ with the topology and ordering inherited from $\mathbb{R}$. The forward and backward jump operators $\sigma, \rho: \mathbb{T} \rightarrow \mathbb{T}$ and the graininess $\mu: \mathbb{T} \rightarrow \mathbb{R}^{+}$ are defined, respectively, by

$$
\begin{gathered}
\sigma(t):=\inf \{s \in \mathbb{T}: s>t\}, \\
\rho(t):=\sup \{s \in \mathbb{T}: s<t\}, \\
\mu(t):=\sigma(t)-t .
\end{gathered}
$$

The point $t \in \mathbb{T}$ is called left-dense, left-scattered, right-dense, or right-scattered if $\rho(t)=t, \rho(t)<t, \sigma(t)=t$, or $\sigma(t)>t$, respectively. Points that are right-dense and left-dense at the same time are called dense. If $\mathbb{T}$ has a left-scattered maximum $m$, define $\mathbb{T}^{k}=\mathbb{T} \backslash\{m\}$; otherwise, $\mathbb{T}^{k}=\mathbb{T}$.

Definition 2 (see $[24,26])$. A vector function $f: \mathbb{T} \rightarrow \mathbb{R}^{n}$ is rd-continuous provided it is continuous at each right-dense point in $\mathbb{T}$ and has a left-sided limit at each left-dense point in $\mathbb{T}$. The set of rd-continuous functions $f: \mathbb{T} \rightarrow \mathbb{R}^{n}$ will be denoted by $C_{\text {rd }}(\mathbb{T})=C_{\text {rd }}\left(\mathbb{T}, \mathbb{R}^{n}\right)$.

Definition 3 (see $[24,26]$ ). For a function $f: \mathbb{T} \rightarrow \mathbb{R}$ (the range $\mathbb{R}$ of $f$ may be actually replaced by Banach space), the (delta) derivative is defined by

$$
f^{\Delta}=\frac{f(\sigma(t))-f(t)}{\sigma(t)-t},
$$

if $f$ is continuous at $t$ and $t$ is right-scattered. If $t$ is not rightscattered, then the derivative is defined by

$$
f^{\Delta}=\lim _{s \rightarrow t} \frac{f(\sigma(t))-f(s)}{\sigma(t)-s}=\lim _{t \rightarrow s} \frac{f(t)-f(s)}{t-s},
$$

provided that this limit exists.

Definition 4 (see $[23,24,26])$. If $F^{\Delta}(t)=f(t)$, then one defines the delta integral by

$$
\int_{a}^{t} f(s) \Delta s=F(t)-F(a)
$$

Definition 5 (see $[23,24]$ ). If $a \in \mathbb{T}$, sup $\mathbb{T}=\infty$, and $f$ is rdcontinuous on $[a, \infty)_{\mathbb{T}}$, then we define the improper integral by

$$
\int_{a}^{\infty} f(s) \Delta s:=\lim _{b \rightarrow \infty} \int_{a}^{b} f(s) \Delta s,
$$

provided that this limit exists, and one says that the improper integral converges in this case. If this limit does not exist, then one says that the improper integral diverges.

Definition 6 (see $[24,26]$ ). A function $p: \mathbb{T} \rightarrow \mathbb{R}$ is called regressive if $1+\mu(t) p(t) \neq 0$ for all $t \in \mathbb{T}^{k}$, where $\mu(t)=$ $\sigma(t)-t$ is the graininess function. The set of all regressive rd-continuous functions $f: \mathbb{T} \rightarrow \mathbb{R}$ is denoted by $\mathscr{R}$, while the set $\mathscr{R}^{+}$is given by $\{f \in \mathscr{R}: 1+\mu(t) f(t)>0\}$ for all $t \in \mathbb{T}$. Let $p \in \mathscr{R}$. The exponential function is defined by

$$
e_{p}(t, s)=\exp \left\{\int_{s}^{t} \xi_{\mu(\tau)}(p(\tau)) \Delta \tau\right\} \quad \text { for } s, t \in \mathbb{T}
$$

with the cylinder transformation

$$
\xi_{h}(z)= \begin{cases}\frac{\log (1+h z)}{h} & \text { if } h \neq 0, \\ z & \text { if } h=0 .\end{cases}
$$

Definition 7 (see $[18,24]$ ). For each $t \in \mathbb{R}$, let $N$ be a neighborhood of $t$. Then one defined the generalized derivative (or Dini derivative) $D^{+} u^{\Delta}(t)$ to mean that, given $\varepsilon>0$, there exists a right neighborhood $N(\varepsilon) \subset N$ of $\epsilon$ such that

$$
\frac{u(\sigma(t))-u(s)}{\sigma(t)-s}<D^{+} u^{\Delta}(t)+\epsilon
$$

for each $s \in N(\epsilon), s>t$.

In case $t$ is right-scattered and $u(t)$ is continuous at $t$, this reduces to

$$
D^{+} u^{\Delta}(t)=\frac{u(\sigma(t))-u(t)}{\sigma(t)-t}
$$

where the upper right Dini derivative is defined as

$$
D^{+} u(t)=\lim _{h \rightarrow 0^{+}} \sup \frac{u(t+h)-u(t)}{h} .
$$


Definition 8 (see [18]). One says that a time scale $\mathbb{T}$ is periodic if there exists $p>0$ such that if $t \in \mathbb{T}$, then $t \pm p \in \mathbb{T}$. For $\mathbb{T} \neq \mathbb{R}$, the smallest positive $p$ is called the period of the time scale. Let $\mathbb{T} \neq \mathbb{R}$ be a periodic time scale with period $p$. We say that the function $f: \mathbb{T} \rightarrow \mathbb{R}$ is $(\omega / 2)$-antiperiodic if there exists a natural number $n$ such that $\omega / 2=n p, f(t+$ $\omega / 2)=-f(t)$ for all $t \in \mathbb{T}$ and $\omega / 2$ is the smallest number such that $f(t+\omega / 2)=-f(t)$. If $\mathbb{T}=\mathbb{R}$, one says that $f$ is $(\omega / 2)$-antiperiodic if $\omega / 2$ is the smallest positive number such that $f(t+\omega / 2)=-f(t)$ for all $t \in \mathbb{T}$.

Lemma 9 (see $[23,24,26])$. Assume that $p, q \in \mathscr{R}$. Then
(a) $e_{0}(t, s) \equiv 1$ and $e_{p}(t, t) \equiv 1$;
(b) $e_{p}(\sigma(t), s)=(1+\mu(t) p(t)) e_{p}(t, s)$;
(c) $1 / e_{p}(t, s)=e_{\odot p}(t, s)$, where $\Theta p(t):=-p(t) /(1+$ $\mu(t) p(t))$
(d) $e_{p}(t, s) e_{p}(s, r)=e_{p}(t, r)$;
(e) $e_{p}^{\Delta}(\cdot, s)=p e_{p}(\cdot, s)$.

Lemma 10 (see [28]). If $p \in \mathscr{R}^{+}$, then

$$
0<e_{p}(t, s) \leq \exp \left(\int_{s}^{t}(p(u)) \Delta u\right) .
$$

Lemma 11 (see $[23,24,26]$ ). Assume that $f, g: \mathbb{T} \rightarrow \mathbb{R}$ are delta differentiable at $t \in T^{k}$. Then

$$
\begin{aligned}
(f g)^{\Delta}(t) & =f^{\Delta}(t) g(t)+f(\sigma(t)) g^{\Delta}(t) \\
& =f(t) g^{\Delta}(t)+f^{\Delta}(t) g(\sigma(t)) .
\end{aligned}
$$

Lemma 12 (see $[18,24,27])$. Let $t_{1}, t_{2} \in[0, \omega]_{\mathbb{T}}$. If $x: \mathbb{T} \rightarrow \mathbb{R}$ is $\omega$-periodic, then

$$
\begin{aligned}
& x(t) \leq x\left(t_{1}\right)+\int_{0}^{\omega}\left|x^{\Delta}(s)\right| \Delta s, \\
& x(t) \geq x\left(t_{2}\right)-\int_{0}^{\omega}\left|x^{\Delta}(s)\right| \Delta s .
\end{aligned}
$$

Definition 13 (see [18]). The antiperiodic solution $x^{*}(t)=$ $\left(x_{1}^{*}(t), \ldots, x_{n}^{*}(t)\right)$ of system (3) with initial value $\varphi^{*}(t)=$ $\left(\varphi_{1}^{*}(t), \ldots, \varphi_{n}^{*}(t)\right)$ is said to be globally exponentially stable if there exist positive constants $\lambda$ and $M=M(\lambda) \geq 1$, for any solution $x(t)=\left(x_{1}(t), \ldots, x_{n}(t)\right)^{T}$ of system (3) with the initial value $\varphi(t)=\left(\varphi_{1}(t), \ldots, \varphi_{n}(t)\right)^{T}$, such that

$$
\begin{array}{r}
\left|x_{i}(t)-x_{i}^{*}(t)\right| \leq M(\lambda) e_{\odot \lambda}(t, \alpha)\left\|\varphi-\varphi^{*}\right\|_{\infty}, \\
\forall t \in(0, \infty)_{\mathbb{T}}, \quad i=1,2, \ldots, n,
\end{array}
$$

where

$$
\begin{array}{r}
\left\|\varphi-\varphi^{*}\right\|_{\infty}=\sup _{-\infty<s<0} \max _{1 \leq i \leq n}\left|\varphi_{i}(s)-\varphi_{i}^{*}(s)\right|, \\
\alpha \in(-\infty, 0]_{\mathbb{V}} .
\end{array}
$$

The following fixed point theorem of coincidence degree is crucial in the arguments of our main results.
Lemma 14 (see $[18,29])$. Let $\mathbb{X}, \mathbb{Y}$ be two Banach spaces and let $\Omega \subset \mathbb{X}$ be open bounded and symmetric with $0 \in \Omega$. Suppose that $L: D(L) \subset \mathbb{X} \rightarrow \mathbb{Y}$ is a linear Fredholm operator of index zero with $D(L) \cap \bar{\Omega} \neq \emptyset$ and $N: \bar{\Omega} \rightarrow \mathbb{Y}$ is L-compact. Further, one also assumes that

(H) $L x-N x \neq \lambda(-L x-N(-x))$ for all $x \in D(L) \cap \partial \Omega, \lambda \epsilon$ $(0,1]$.

Then $L x=N x$ has at least one solution on $D(L) \cap \bar{\Omega}$.

Lemma 15 (mean value theorem, $[6,30]$ ). Let $f$ be a continuous function on $[a, b]_{\mathbb{T}}$ which is $\Delta$-differentiable on $[a, b)_{\mathbb{T}}$, and then there exist $\xi, \tau \in[a, b)_{\mathbb{T}}$ such that

$$
f^{\Delta}(\xi)(b-a) \leq f(b)-f(a) \leq f^{\Delta}(\tau)(b-a) .
$$

\section{Existence of Antiperiodic Solutions}

In this section, by using fixed point theorem of coincidence degree, we will study the existence of at least one antiperiodic solution for system (3).

Theorem 16. Assume that $\left(H_{1}\right)-\left(H_{7}\right)$ hold. Suppose further that $E_{i}>0, i=1,2, \ldots, n$. Then system (3) has at least one $(\omega / 2)$-antiperiodic solution.

Proof. Let $C^{k}\left(\left[0, \omega ; t_{1}, t_{2}, \ldots, t_{q}, t_{q+1}, \ldots, t_{2 q}\right]_{\mathbb{T}}, \mathbb{R}^{n}\right)=\{x:$ $[0, \omega]_{\mathbb{T}} \rightarrow \mathbb{R}^{n} \mid x^{(k)}(t)$ be a piecewise continuous map with first-class discontinuous points in $[0, \omega]_{\mathbb{T}} \cap\left\{t_{k}: k \in \mathbb{N}\right\}$ and at each discontinuous point it is continuous on the left $\}, k=$ 0,1 . Let

$$
\begin{gathered}
\mathbb{X}=\left\{x \in C\left(\left[0, \omega ; t_{1}, t_{2}, \ldots, t_{q}, t_{q+1}, \ldots, t_{2 q}\right]_{\mathbb{T}}, \mathbb{R}^{n}\right)\right. \\
\left.: x\left(t+\frac{\omega}{2}\right)=-x(t) \forall t \in\left[0, \frac{\omega}{2}\right]_{\mathbb{T}}\right\}, \\
\mathbb{Y}=\mathbb{X} \times \mathbb{R}^{n \times q}
\end{gathered}
$$

be two Banach spaces equipped with the norms

$$
\|x\|_{\mathbb{X}}=\sum_{i=1}^{n}\left|x_{i}\right|_{0}, \quad\|y\|_{\mathbb{Y}}=\|x\|_{\mathbb{X}}+\|z\| \quad \forall x \in \mathbb{X}, z \in \mathbb{R}^{n \times q},
$$

in which $\left|x_{i}\right|_{0}=\max _{t \in[0, \omega]_{\mathbb{T}}}\left|x_{i}(t)\right|, i=1,2, \ldots, n$ and $\|\cdot\|$ is any norm of $\mathbb{R}^{n \times q}$. Set

$$
\begin{gathered}
L: \operatorname{Dom} L \cap \mathbb{X} \longrightarrow \mathbb{Y}, \\
x \longrightarrow\left(x^{\Delta}, \Delta x\left(t_{1}\right), \Delta x\left(t_{2}\right), \ldots, \Delta x\left(t_{q}\right)\right),
\end{gathered}
$$

where

$$
\begin{aligned}
\operatorname{Dom} L=\left\{x \in C^{1}\left(\left[0, \omega ; t_{1}, t_{2}, \ldots, t_{2 q}\right]_{\mathbb{T}}, \mathbb{R}^{n}\right)\right. \\
\left.: x\left(t+\frac{\omega}{2}\right)=-x(t) \forall t \in\left[0, \frac{\omega}{2}\right]_{\mathbb{V}}\right\},
\end{aligned}
$$


and $N: \mathbb{X} \rightarrow \mathbb{Y}$ and

$N x$

$$
\begin{aligned}
= & \left(\left(\begin{array}{c}
A_{1}(t) \\
A_{2}(t) \\
\vdots \\
A_{n}(t)
\end{array}\right),\left(\begin{array}{c}
I_{11}\left(x_{1}\left(t_{1}\right)\right) \\
I_{21}\left(x_{2}\left(t_{1}\right)\right) \\
\vdots \\
I_{n 1}\left(x_{n}\left(t_{1}\right)\right)
\end{array}\right),\right. \\
& \left.\left(\begin{array}{c}
I_{12}\left(x_{1}\left(t_{2}\right)\right) \\
I_{22}\left(x_{2}\left(t_{2}\right)\right) \\
\vdots \\
I_{n 2}\left(x_{n}\left(t_{2}\right)\right)
\end{array}\right), \ldots,\left(\begin{array}{c}
I_{1 q}\left(x_{1}\left(t_{q}\right)\right) \\
I_{2 q}\left(x_{2}\left(t_{q}\right)\right) \\
\vdots \\
I_{n q}\left(x_{n}\left(t_{q}\right)\right)
\end{array}\right)\right),
\end{aligned}
$$

where

$$
\begin{aligned}
A_{i}(t)= & -\alpha_{i}\left(x_{i}(t)\right) \\
& \times\left[\beta_{i}\left(x_{i}(t)\right)-\sum_{j=1}^{n} a_{i j}(t) f_{j}\left(x_{j}(t)\right)\right. \\
& \quad-\sum_{j=1}^{n} b_{i j}(t) g_{j}\left(x_{j}\left(t-\tau_{i j}(t)\right)\right) \\
& -\sum_{j=1}^{n} c_{i j}(t) \int_{0}^{+\infty} K_{i j}(s) h_{j}\left(x_{j}(t-s)\right) \Delta s \\
& \left.+J_{i}(t)\right],
\end{aligned}
$$

for $i=1,2, \ldots, n$. It is easy to see that

$$
\begin{aligned}
& \operatorname{Ker} L=\{0\}, \\
& \operatorname{Im} L=\left\{y=\left(g, c_{1}, \ldots, c_{q}\right) \in \mathbb{Y}: \int_{0}^{\omega} g(s) \Delta s=0\right\} \equiv \mathbb{V} .
\end{aligned}
$$

Thus, $\operatorname{dim} \operatorname{Ker} L=0=\operatorname{codim} \operatorname{Im} L$, and $L$ is a linear Fredholm operator of index zero.

Define the continuous projector $P: \mathbb{X} \rightarrow \operatorname{Ker} L$ and the averaging projector $Q: \mathbb{Y} \rightarrow \mathbb{Y}$ by

$$
\begin{gathered}
P x=\int_{0}^{\omega} x(s) \Delta s=0, \\
Q y=Q\left(g, c_{1}, \ldots, c_{q}\right)=\left(\frac{1}{\omega} \int_{0}^{\omega} g(s) \Delta s, 0, \ldots, 0\right) .
\end{gathered}
$$

Hence, $\operatorname{Im} P=\operatorname{Ker} L$ and $\operatorname{Ker} Q=\operatorname{Im} L=\operatorname{Im}(I-Q)$. Denoting by $L_{p}^{-1}: \operatorname{Im} L \rightarrow \operatorname{Dom}(L) \cap \operatorname{Ker} P$ the inverse of $\left.L\right|_{\text {Dom }(L) \cap K e r P}$, we have

$$
L_{p}^{-1} y=\int_{0}^{t} g(s) \Delta s+\sum_{t>t_{k}} c_{k}-\frac{1}{2} \int_{0}^{\omega / 2} g(s) \Delta s-\frac{1}{2} \sum_{k=1}^{q} c_{k},
$$

in which $c_{q+i}=-c_{i}$ for all $1 \leq i \leq q$.
Similar to [24], it is not difficult to show that $Q N(\bar{\Omega})$, $L_{p}^{-1}(I-Q)(\bar{\Omega})$ are relatively compact for any open bounded set $\Omega \subset \mathbb{X}$. Therefore, $N$ is $L$-compact on $\bar{\Omega}$ for any open bounded set $\Omega \subset \mathbb{X}$.

In order to apply Lemma 14, we need to find an appropriate open bounded subset $\Omega$ in $\mathbb{X}$. Corresponding to the operator equation $L x-N x=\lambda(-L x-N(-x)), \lambda \in(0,1]$, we have

$$
\begin{array}{r}
x_{i}^{\Delta}(t)=\frac{1}{1+\lambda} G_{i}(t, x)-\frac{\lambda}{1+\lambda} G_{i}(t,-x), \\
t \in \mathbb{T}^{+}, \quad t \neq t_{k}, \quad k \in \mathbb{N}, \\
\Delta x_{i}\left(t_{k}\right)=\frac{1}{1+\lambda} I_{i k}\left(x_{i}\left(t_{k}\right)\right)-\frac{\lambda}{1+\lambda} I_{i k}\left(-x_{i}\left(t_{k}\right)\right), \\
\quad i=1,2, \ldots, n,
\end{array}
$$

where

$$
\begin{aligned}
G_{i}(t, x)= & -\alpha_{i}\left(x_{i}(t)\right) \\
& \times\left[\beta_{i}\left(x_{i}(t)\right)-\sum_{j=1}^{n} a_{i j}(t) f_{j}\left(x_{j}(t)\right)\right. \\
& -\sum_{j=1}^{n} b_{i j}(t) g_{j}\left(x_{j}\left(t-\tau_{i j}(t)\right)\right) \\
& -\sum_{j=1}^{n} c_{i j}(t) \int_{0}^{+\infty} K_{i j}(s) h_{j}\left(x_{j}(t-s)\right) \Delta s \\
& \left.+J_{i}(t)\right],
\end{aligned}
$$$$
G_{i}(t,-x)=-\alpha_{i}\left(-x_{i}(t)\right)
$$$$
\times\left[\beta_{i}\left(-x_{i}(t)\right)-\sum_{j=1}^{n} a_{i j}(t) f_{j}\left(-x_{j}(t)\right)\right.
$$$$
-\sum_{j=1}^{n} b_{i j}(t) g_{j}\left(-x_{j}\left(t-\tau_{i j}(t)\right)\right)
$$$$
-\sum_{j=1}^{n} c_{i j}(t) \int_{0}^{+\infty} K_{i j}(s) h_{j}\left(-x_{j}(t-s)\right) \Delta s
$$$$
\left.+J_{i}(t)\right] \text {, }
$$

for $i=1,2, \ldots, n$. 
6

Abstract and Applied Analysis

Set $t_{0}=t_{0}^{+}=0, t_{2 q+1}=\omega$. Then, by (31), $\left(\mathrm{H}_{2}\right),\left(\mathrm{H}_{3}\right),\left(\mathrm{H}_{5}\right), \quad$ Integrating (31) from 0 to $\omega$, we have by (33) $\left(\mathrm{H}_{6}\right)$, and Lemma 15, we obtain that

$$
\begin{aligned}
& \int_{0}^{\omega}\left|x_{i}^{\Delta}(t)\right| \Delta t \\
& =\sum_{k=1}^{2 q+1} \int_{t_{k-1}^{+}}^{t_{k}}\left|x_{i}^{\Delta}(t)\right| \Delta t+\sum_{k=1}^{2 q}\left|\Delta x_{i}\left(t_{k}\right)\right| \\
& \leq \int_{0}^{\omega}\left|\frac{1}{1+\lambda} G_{i}(t, x)-\frac{\lambda}{1+\lambda} G_{i}(t,-x)\right| \Delta t \\
& +\sum_{k=1}^{2 q}\left|\frac{1}{1+\lambda} I_{i k}\left(x_{i}\left(t_{k}\right)\right)-\frac{\lambda}{1+\lambda} I_{i k}\left(-x_{i}\left(t_{k}\right)\right)\right| \\
& \leq\left(\frac{1}{1+\lambda}+\frac{\lambda}{1+\lambda}\right) \int_{0}^{\omega} \max \left\{\left|G_{i}(t, x)\right|,\left|G_{i}(t,-x)\right|\right\} \Delta t \\
& +\sum_{k=1}^{2 q} \frac{1}{1+\lambda}\left|I_{i k}\left(x_{i}\left(t_{k}\right)\right)-I_{i k}(0)\right| \\
& +\sum_{k=1}^{2 q} \frac{\lambda}{1+\lambda}\left|I_{i k}\left(-x_{i}\left(t_{k}\right)\right)-I_{i k}(0)\right|+\sum_{k=1}^{2 q}\left|I_{i k}(0)\right| \\
& \leq \alpha_{i}^{M}\left(\int_{0}^{\omega}\left|\beta_{i}\left(x_{i}(t)\right)\right| \Delta t+\sum_{j=1}^{n} a_{i j}^{M} \int_{0}^{\omega}\left|f_{j}\left(x_{j}(t)\right)\right| \Delta t\right. \\
& +\sum_{j=1}^{n} b_{i j}^{M} \int_{0}^{\omega}\left|g_{j}\left(x_{j}\left(t-\tau_{i j}(t)\right)\right)\right| \Delta t \\
& +\sum_{j=1}^{n} c_{i j}^{M} \int_{0}^{\infty}\left|K_{i j}(s)\right| \Delta s \\
& \left.\times \int_{0}^{\omega}\left|h_{j}\left(x_{j}(t-s)\right)\right| \Delta t+J_{i}^{M} \omega\right) \\
& +\sum_{k=1}^{2 q} \rho_{i k}\left|x_{i}\right|_{0}+\sum_{k=1}^{2 q}\left|I_{i k}(0)\right| \\
& \leq \alpha_{i}^{M} \omega\left(\delta_{i}\left|x_{i}\right|_{0}+\sum_{j=1}^{n} a_{i j}^{M} f_{j}^{M}+\sum_{j=1}^{n} b_{i j}^{M} g_{j}^{M}\right. \\
& \left.+\sum_{j=1}^{n} c_{i j}^{M} h_{j}^{M} \int_{0}^{\infty}\left|K_{i j}(s)\right| \Delta s+J_{i}^{M}\right) \\
& +\sum_{k=1}^{2 q} \rho_{i k}\left|x_{i}\right|_{0}+\sum_{k=1}^{2 q}\left|I_{i k}(0)\right| \\
& =\alpha_{i}^{M} \omega\left(\delta_{i}\left|x_{i}\right|_{0}+B_{i}+J_{i}^{M}\right)+\sum_{k=1}^{2 q} \rho_{i k}\left|x_{i}\right|_{0}+\sum_{k=1}^{2 q}\left|I_{i k}(0)\right|, \\
& i=1,2, \ldots, n \text {. }
\end{aligned}
$$

$$
\begin{aligned}
& \left|\int_{0}^{\omega} \alpha_{i}\left(x_{i}(t)\right) \beta_{i}\left(x_{i}(t)\right) \Delta t\right| \\
& =\mid \frac{1}{1+\lambda} \int_{0}^{\omega} \alpha_{i}\left(x_{i}(t)\right) \sum_{j=1}^{n} a_{i j}(t) f_{j}\left(x_{j}(t)\right) \Delta t \\
& -\frac{\lambda}{1+\lambda} \int_{0}^{\omega} \alpha_{i}\left(-x_{i}(t)\right) \sum_{j=1}^{n} a_{i j}(t) f_{j}\left(-x_{j}(t)\right) \Delta t \\
& +\frac{1}{1+\lambda} \int_{0}^{\omega} \alpha_{i}\left(x_{i}(t)\right) \sum_{j=1}^{n} b_{i j}(t) g_{j}\left(x_{j}\left(t-\tau_{i j}(t)\right)\right) \Delta t \\
& -\frac{\lambda}{1+\lambda} \int_{0}^{\omega} \alpha_{i}\left(-x_{i}(t)\right) \sum_{j=1}^{n} b_{i j}(t) g_{j}\left(-x_{j}\left(t-\tau_{i j}(t)\right)\right) \Delta t \\
& +\frac{\lambda}{1+\lambda} \sum_{k=1}^{2 q} I_{i k}\left(x_{i}\left(t_{k}\right)\right)-\frac{\lambda}{1+\lambda} \sum_{k=1}^{2 q} I_{i k}\left(-x_{i}\left(t_{k}\right)\right) \\
& +\frac{1}{1+\lambda} \int_{0}^{\omega} \alpha_{i}\left(x_{i}(t)\right) \\
& \times \sum_{j=1}^{n} c_{i j}(t) \int_{0}^{\infty} K_{i j}(s) h_{j}\left(x_{j}(t-s)\right) \Delta s \Delta t \\
& -\frac{\lambda}{1+\lambda} \int_{0}^{\omega} \alpha_{i}\left(-x_{i}(t)\right) \\
& \times \sum_{j=1}^{n} c_{i j}(t) \int_{0}^{\infty} K_{i j}(s) h_{j}\left(-x_{j}(t-s)\right) \Delta s \Delta t \\
& -\frac{1}{1+\lambda} \int_{0}^{\omega} \alpha_{i}\left(x_{i}(t)\right) J_{i}(t) \Delta t \\
& +\frac{\lambda}{1+\lambda} \int_{0}^{\omega} \alpha_{i}\left(x_{i}(t)\right) J_{i}(t) \Delta t \\
& \leq \alpha_{i}^{M} \omega\left(\sum_{j=1}^{n} a_{i j}^{M} f_{j}^{M}+\sum_{j=1}^{n} b_{i j}^{M} g_{j}^{M}\right. \\
& \left.+\sum_{j=1}^{n} c_{i j}^{M} h_{j}^{M} \int_{0}^{\infty}\left|K_{i j}(s)\right| \Delta s+J_{i}^{M}\right) \\
& +\left(\frac{1}{1+\lambda}+\frac{\lambda}{1+\lambda}\right) \\
& \times \max \left\{\left|\sum_{k=1}^{2 q} I_{i k}\left(x_{i}\left(t_{k}\right)\right)\right|,\left|\sum_{k=1}^{2 q} I_{i k}\left(-x_{i}\left(t_{k}\right)\right)\right|\right\} \\
& \leq \alpha_{i}^{M} \omega\left(\sum_{j=1}^{n} a_{i j}^{M} f_{j}^{M}+\sum_{j=1}^{n} b_{i j}^{M} g_{j}^{M}\right.
\end{aligned}
$$


Abstract and Applied Analysis

7

$$
\begin{gathered}
\left.+\sum_{j=1}^{n} c_{i j}^{M} h_{j}^{M} \int_{0}^{\infty}\left|K_{i j}(s)\right| \Delta s+J_{i}^{M}\right) \\
+\max \left\{\left|\sum_{k=1}^{2 q} I_{i k}\left(x_{i}\left(t_{k}\right)\right)-I_{i k}(0)\right|,\right. \\
\leq \alpha_{i}^{M} \omega\left(B_{i}+J_{i}^{M}\right)+\sum_{k=1}^{2 q} \rho_{i k}\left|x_{i 0}\right|+\sum_{k=1}^{2 q} I_{i k}(0), \\
i=1,2, \ldots, n .
\end{gathered}
$$

In view of $(34),\left(\mathrm{H}_{3}\right)$, and Lemma 15 , we get

$$
\begin{aligned}
\left|\int_{0}^{\omega} \alpha_{i}\left(x_{i}(t)\right) x_{i}(t) \Delta t\right| \leq & \frac{1}{\rho_{i}}\left|\int_{0}^{\omega} \alpha_{i}\left(x_{i}(t)\right) \beta_{i}\left(x_{i}(t)\right) \Delta t\right| \\
\leq & \frac{1}{\rho_{i}} \alpha_{i}^{M} \omega\left(B_{i}+J_{i}^{M}\right) \\
& +\frac{1}{\rho_{i}}\left(\sum_{k=1}^{2 q} \rho_{i k}\left|x_{i 0}\right|+\sum_{k=1}^{2 q} I_{i k}(0)\right),
\end{aligned}
$$

for $i=1,2, \ldots, n$. In addition, from Lemma 12 , for any $\xi_{i}, \eta_{i} \in$ $[0, \omega]_{\mathbb{T}}$, we have

$$
\begin{aligned}
\int_{0}^{\omega} \alpha_{i}\left(x_{i}(t)\right) x_{i}(t) \Delta t \leq & \int_{0}^{\omega} \alpha_{i}\left(x_{i}(t)\right) x_{i}\left(\xi_{i}\right) \Delta t \\
& +\int_{0}^{\omega} \alpha_{i}\left(x_{i}(t)\right)\left(\int_{0}^{\omega}\left|x_{i}^{\Delta}(t)\right| \Delta t\right) \Delta t,
\end{aligned}
$$

$$
\begin{aligned}
\int_{0}^{\omega} \alpha_{i}\left(x_{i}(t)\right) x_{i}(t) \Delta t \geq & \int_{0}^{\omega} \alpha_{i}\left(x_{i}(t)\right) x_{i}\left(\eta_{i}\right) \Delta t \\
& -\int_{0}^{\omega} \alpha_{i}\left(x_{i}(t)\right)\left(\int_{0}^{\omega}\left|x_{i}^{\Delta}(t)\right| \Delta t\right) \Delta t,
\end{aligned}
$$

where $i=1,2, \ldots, n$. Dividing by $\int_{0}^{\omega} \alpha_{i}\left(x_{i}(t)\right) \Delta t$ on the two sides of (36) and (37), respectively, we obtain that

$$
\begin{aligned}
x_{i}\left(\xi_{i}\right) \geq & \frac{1}{\int_{0}^{\omega} \alpha_{i}\left(x_{i}(t)\right) \Delta t} \int_{0}^{\omega} \alpha_{i}\left(x_{i}(t)\right) x_{i}(t) \Delta t \\
& -\int_{0}^{\omega}\left|x_{i}^{\Delta}(t)\right| \Delta t, \\
x_{i}\left(\eta_{i}\right) \leq & \frac{1}{\int_{0}^{\omega} \alpha_{i}\left(x_{i}(t)\right) \Delta t} \int_{0}^{\omega} \alpha_{i}\left(x_{i}(t)\right) x_{i}(t) \Delta t \\
& +\int_{0}^{\omega}\left|x_{i}^{\Delta}(t)\right| \Delta t,
\end{aligned}
$$

where $i=1,2, \ldots, n$.
Let $\bar{t}_{i}, \underline{t}_{i} \in[0, \omega]_{\mathbb{T}}$ such that $x_{i}\left(\bar{t}_{i}\right)=\max _{t \in[0, \omega]_{\mathbb{T}}} x_{i}(t)$, $x_{i}\left(\underline{t}_{i}\right)=\min _{t \in[0, \omega]_{\mathbb{T}}} x_{i}(t)$, by the arbitrariness of $\xi_{i}, \eta_{i}$; we get from (33)-(39) that

$$
\begin{aligned}
& x_{i}\left(\underline{t}_{i}\right) \geq \frac{1}{\int_{0}^{\omega} \alpha_{i}\left(x_{i}(t)\right) \Delta t} \int_{0}^{\omega} \alpha_{i}\left(x_{i}(t)\right) x_{i}(t) \Delta t \\
& -\int_{0}^{\omega}\left|x_{i}^{\Delta}(t)\right| \Delta t \\
& \geq-\frac{1}{\int_{0}^{\omega} \alpha_{i}\left(x_{i}(t)\right) \Delta t}\left|\int_{0}^{\omega} \alpha_{i}\left(x_{i}(t)\right) x_{i}(t) \Delta t\right| \\
& -\int_{0}^{\omega}\left|x_{i}^{\Delta}(t)\right| \Delta t \\
& \geq-\frac{1}{\rho_{i} \alpha_{i}^{m}} \alpha_{i}^{M}\left(B_{i}+J_{i}^{M}\right) \\
& -\alpha_{i}^{M} \omega\left(\delta_{i}\left|x_{i}\right|_{0}+B_{i}+J_{i}^{M}\right) \\
& -\left(\frac{1}{\rho_{i} \alpha_{i}^{m} \omega}+1\right)\left(\sum_{k=1}^{2 q} \rho_{i k}\left|x_{i}\right|_{0}+\sum_{k=1}^{2 q}\left|I_{i k}(0)\right|\right) \\
& =-\left(\frac{\alpha_{i}^{M}}{\rho_{i} \alpha_{i}^{m}}+\alpha_{i}^{M} \omega\right)\left(B_{i}+J_{i}^{M}\right)-\alpha_{i}^{M} \delta_{i} \omega\left|x_{i}\right|_{0} \\
& -\left(\frac{1}{\rho_{i} \alpha_{i}^{m} \omega}+1\right)\left(\sum_{k=1}^{2 q} \rho_{i k}\left|x_{i}\right|_{0}+\sum_{k=1}^{2 q}\left|I_{i k}(0)\right|\right), \\
& i=1,2, \ldots, n \text {, } \\
& x_{i}\left(\bar{t}_{i}\right) \leq \frac{1}{\int_{0}^{\omega} \alpha_{i}\left(x_{i}(t)\right) \Delta t} \int_{0}^{\omega} \alpha_{i}\left(x_{i}(t)\right) x_{i}(t) \Delta t \\
& +\int_{0}^{\omega}\left|x_{i}^{\Delta}(t)\right| \Delta t \\
& \leq \frac{1}{\int_{0}^{\omega} \alpha_{i}\left(x_{i}(t)\right) \Delta t}\left|\int_{0}^{\omega} \alpha_{i}\left(x_{i}(t)\right) x_{i}(t) \Delta t\right| \\
& +\int_{0}^{\omega}\left|x_{i}^{\Delta}(t)\right| \Delta t \\
& \leq \frac{1}{\rho_{i} \alpha_{i}^{m}} \alpha_{i}^{M}\left(B_{i}+J_{i}^{M}\right)+\alpha_{i}^{M} \omega\left(\delta_{i}\left|x_{i}\right|_{0}+B_{i}+J_{i}^{M}\right) \\
& +\left(\frac{1}{\rho_{i} \alpha_{i}^{m} \omega}+1\right)\left(\sum_{k=1}^{2 q} \rho_{i k}\left|x_{i}\right|_{0}+\sum_{k=1}^{2 q}\left|I_{i k}(0)\right|\right) \\
& =\left(\frac{\alpha_{i}^{M}}{\rho_{i} \alpha_{i}^{m}}+\alpha_{i}^{M} \omega\right)\left(B_{i}+J_{i}^{M}\right)+\alpha_{i}^{M} \delta_{i} \omega\left|x_{i}\right|_{0} \\
& +\left(\frac{1}{\rho_{i} \alpha_{i}^{m} \omega}+1\right)\left(\sum_{k=1}^{2 q} \rho_{i k}\left|x_{i}\right|_{0}+\sum_{k=1}^{2 q}\left|I_{i k}(0)\right|\right), \\
& i=1,2, \ldots, n \text {. }
\end{aligned}
$$


Thus, we have from (40) that

$$
\begin{aligned}
&\left|x_{i}\right|_{0}= \max _{t \in[0, \omega]_{\mathbb{T}}}\left|x_{i}(t)\right| \\
& \leq\left(\frac{\alpha_{i}^{M}}{\rho_{i} \alpha_{i}^{m}}+\alpha_{i}^{M} \omega\right)\left(B_{i}+J_{i}^{M}\right)+\alpha_{i}^{M} \delta_{i} \omega\left|x_{i}\right|_{0} \\
&+\left(\frac{1}{\rho_{i} \alpha_{i}^{m} \omega}+1\right)\left(\sum_{k=1}^{2 q} \rho_{i k}\left|x_{i}\right|_{0}+\sum_{k=1}^{2 q}\left|I_{i k}(0)\right|\right), \\
& i=1,2, \ldots, n .
\end{aligned}
$$

From (41), we have

$$
\begin{aligned}
& \rho_{i} \alpha_{i}^{m} \omega\left|x_{i}\right|_{0} \leq \alpha_{i}^{M} \omega\left(1+\rho_{i} \alpha_{i}^{m} \omega\right)\left(B_{i}+J_{i}^{M}\right) \\
&+\rho_{i} \alpha_{i}^{M} \alpha_{i}^{m} \delta_{i} \omega^{2}\left|x_{i}\right|_{0}+\left(1+\rho_{i} \alpha_{i}^{m} \omega\right) \\
& \times\left(\sum_{k=1}^{2 q} \rho_{i k}\left|x_{i}\right|_{0}+\sum_{k=1}^{2 q}\left|I_{i k}(0)\right|\right), \\
& i=1,2, \ldots, n .
\end{aligned}
$$

Then, by the assumption of Theorem 16 and (42), we have

$$
\left|x_{i}\right|_{0} \leq \frac{D_{i}}{E_{i}}:=M_{i}, \quad i=1,2, \ldots, n .
$$

Let

$$
M=\sum_{i=1}^{n} M_{i}+1 .
$$

Clearly, $M$ is independent of $\lambda$. Then take

$$
\Omega=\left\{x \in \mathbb{X}:\|x\|_{\mathbb{X}}<M\right\} .
$$

It is clear that $\Omega$ satisfies all the requirements in Lemma 14 and condition $(\mathrm{H})$ is satisfied. In view of all the discussions above, we conclude from Lemma 14 that system (3) has at least one $(\omega / 2)$-antiperiodic solution. This completes the proof.

\section{Global Exponential Stability of Antiperiodic Solutions}

In this section, we will construct some suitable Lyapunov functions to study the global exponential stability of antiperiodic solutions of system (3).

Theorem 17. Assume that $\left(H_{1}\right)-\left(H_{7}\right)$ hold. Suppose further the following.

$\left(\mathrm{H}_{8}\right)$ The impulsive operators $I_{i k}\left(x_{i}(t)\right)$ satisfy

$$
\begin{gathered}
I_{i k}\left(x_{i}(t)\right)=-\gamma_{i k} x_{i}\left(t_{k}\right), \\
0 \leq \gamma_{i k} \leq 2, \quad i=1,2, \ldots, n, \quad k \in \mathbb{N} .
\end{gathered}
$$

$\left(\mathrm{H}_{9}\right)$ For $t \in(0, \infty)_{\mathbb{T}}$, there exist constants $\epsilon>0$ and $\eta>0$ such that

$$
\begin{array}{r}
l_{i}=\epsilon-\alpha_{i}^{l} \rho_{i}+(1+\mu(t) \epsilon)\left(\alpha_{i}^{L} B_{i}+\alpha_{i}^{M} R_{i}\right)<-\eta<0, \\
i=1,2, \ldots, n .
\end{array}
$$

Then the ( $\omega / 2)$-antiperiodic solution of system (3) is globally exponentially stable.
Proof. According to Theorem 16 and its proof, we know that system (3) has an $(\omega / 2)$-antiperiodic solution $x^{*}=$ $\left(x_{1}^{*}(t), x_{2}^{*}(t), \ldots, x_{n}^{*}(t)\right)^{T}$ with the initial value $\varphi^{*}=\left(\varphi_{1}^{*}(t)\right.$, $\left.\varphi_{2}^{*}(t), \ldots, \varphi_{n}^{*}(t)\right)^{T}$ and $\left|x_{i}^{*}\right|_{0} \leq M_{i}$, and suppose that $x(t)=$ $\left(x_{1}(t), x_{2}(t), \ldots, x_{n}(t)\right)^{T}$ is an arbitrary solution of system (3) with the initial value $\varphi(t)=\left(\varphi_{1}(t), \varphi_{2}(t), \ldots, \varphi_{n}(t)\right)^{T}$. Set $y_{i}(t)=x_{i}(t)-x_{i}^{*}(t)$. Then it follows from system (3) and $\left(\mathrm{H}_{8}\right)$ that

$$
\begin{aligned}
& y_{i}^{\Delta}(t)=-\left(\alpha_{i}\left(x_{i}(t)\right) \beta_{i}\left(x_{i}(t)\right)-\alpha_{i}\left(x_{i}^{*}(t)\right) \beta_{i}\left(x_{i}^{*}(t)\right)\right) \\
& +\left(\alpha_{i}\left(x_{i}(t)\right) \sum_{j=1}^{n} a_{i j}(t) f_{j}\left(x_{j}(t)\right)\right. \\
& \left.-\alpha_{i}\left(x_{i}^{*}(t)\right) \sum_{j=1}^{n} a_{i j}(t) f_{j}\left(x_{j}^{*}(t)\right)\right) \\
& +\left(\alpha_{i}\left(x_{i}(t)\right) \sum_{j=1}^{n} b_{i j}(t) g_{j}\left(x_{j}\left(t-\tau_{i j}(t)\right)\right)\right. \\
& \left.-\alpha_{i}\left(x_{i}^{*}(t)\right) \sum_{j=1}^{n} b_{i j}(t) g_{j}\left(x_{j}^{*}\left(t-\tau_{i j}(t)\right)\right)\right) \\
& +\left(\alpha_{i}\left(x_{i}(t)\right) \sum_{j=1}^{n} c_{i j}(t) \int_{0}^{+\infty} K_{i j}(s) h_{j}\left(x_{j}(t-s)\right) \Delta s\right. \\
& -\alpha_{i}\left(x_{i}^{*}(t)\right) \\
& \left.\times \sum_{j=1}^{n} c_{i j}(t) \int_{0}^{+\infty} K_{i j}(s) h_{j}\left(x_{j}^{*}(t-s)\right) \Delta s\right), \\
& \Delta y_{i}\left(t_{k}\right)=-\gamma_{i k} y_{i}\left(t_{k}\right), \quad i=1,2, \ldots, n \text {. }
\end{aligned}
$$

In view of the above system and $\left(\mathrm{H}_{2}\right)-\left(\mathrm{H}_{6}\right)$, for $t \epsilon$ $\mathbb{T}^{+}, t \neq t_{k}, k \in \mathbb{N}, i=1,2, \ldots, n$, similar to [6], we have

$$
\begin{aligned}
& D^{+}\left|y_{i}^{\Delta}(t)\right| \\
& \leq-\alpha_{i}^{l}\left|\beta_{i}\left(x_{i}(t)\right)-\beta_{i}\left(x_{i}^{*}(t)\right)\right| \\
&+\alpha_{i}^{L} \sum_{j=1}^{n}\left(a_{i j}^{M} f_{j}^{M}+b_{i j}^{M} g_{j}^{M}+c_{i j}^{M} h_{j}^{M} \int_{0}^{+\infty}\left|K_{i j}(s)\right| \Delta s\right) \\
& \times\left|y_{i}(t)\right|+\alpha_{i}^{M} \sum_{j=1}^{n} a_{i j}^{M} f_{j}^{L}\left|y_{j}(t)\right| \\
&+\alpha_{i}^{M} \sum_{j=1}^{n} b_{i j}^{M} g_{j}^{L}\left|y_{j}\left(t-\tau_{i j}(t)\right)\right| \\
&+\alpha_{i}^{M} \sum_{j=1}^{n} c_{i j}^{M} h_{j}^{L} \int_{0}^{+\infty}\left|K_{i j}(s)\right|\left|y_{j}(t-s)\right| \Delta s
\end{aligned}
$$




$$
\begin{aligned}
\leq & -\alpha_{i}^{l} \rho_{i}\left|y_{i}(t)\right|+\alpha_{i}^{L} B_{i}\left|y_{i}(t)\right|+\alpha_{i}^{M} \sum_{j=1}^{n} a_{i j}^{M} f_{j}^{L}\left|y_{j}(t)\right| \\
& +\alpha_{i}^{M} \sum_{j=1}^{n} b_{i j}^{M} g_{j}^{L}\left|y_{j}\left(t-\tau_{i j}(t)\right)\right| \\
& +\alpha_{i}^{M} \sum_{j=1}^{n} c_{i j}^{M} h_{j}^{L} \int_{0}^{+\infty}\left|K_{i j}(s)\right|\left|y_{j}(t-s)\right| \Delta s .
\end{aligned}
$$

For any $a \in(-\infty, 0]_{\mathbb{T}}$, we consider the following Lyapunov function:

$$
V_{i}(t)=\left|y_{i}(t)\right| e_{\varepsilon}(t, a), \quad i=1,2, \ldots n .
$$

For $t \in \mathbb{T}^{+}, t \neq t_{k}, k \in \mathbb{N}$, calculating the upper right derivative of $V_{i}(t)$ by (48)-(50), we have

$$
\begin{aligned}
D^{+}\left|V_{i}^{\Delta}(t)\right| & \\
= & D^{+}\left|y_{i}^{\Delta}(t)\right| e_{\varepsilon}(\sigma(t), a)+\left|y_{i}(t)\right| e_{\varepsilon}^{\Delta}(t, a) \\
\leq & \epsilon\left|y_{i}(t)\right| e_{\varepsilon}(t, a)+e_{\varepsilon}(\sigma(t), a) \\
& \times\left(-\alpha_{i}^{l} \rho_{i}\left|y_{i}(t)\right|+\alpha_{i}^{L} B_{i}\left|y_{i}(t)\right|+\alpha_{i}^{M} \sum_{j=1}^{n} a_{i j}^{M} f_{j}^{L}\left|y_{j}(t)\right|\right. \\
& +\alpha_{i}^{M} \sum_{j=1}^{n} b_{i j}^{M} g_{j}^{L}\left|y_{j}\left(t-\tau_{i j}(t)\right)\right|+\alpha_{i}^{M} \\
& \left.\times \sum_{j=1}^{n} c_{i j}^{M} h_{j}^{L} \int_{0}^{+\infty}\left|K_{i j}(s)\right|\left|y_{j}(t-s)\right| \Delta s e_{\varepsilon}(\sigma(t), a)\right) \\
=\epsilon V_{i}(t)+(1+\epsilon \mu(t)) & +\left(-\alpha_{i}^{l} \rho_{i}+\alpha_{i}^{L} B_{i}\right) V_{i}(t)+\alpha_{i}^{M} \sum_{j=1}^{n} a_{i j}^{M} f_{j}^{L} V_{j}(t) \\
\quad & +\alpha_{i}^{M} \sum_{j=1}^{n} b_{i j}^{M} g_{j}^{L} V_{j}\left(t-\tau_{i j}(t)\right) e_{\varepsilon}\left(t, t-\tau_{i j}(t)\right)+\alpha_{i}^{M} \\
& \left.\times \sum_{j=1}^{n} c_{i j}^{M} h_{j}^{L} \int_{0}^{+\infty}\left|K_{i j}(s)\right| e_{\varepsilon}(t, t-s) V_{j}(t-s) \Delta s\right),
\end{aligned}
$$

for $i=1,2, \ldots, n$. In addition, for $t \in \mathbb{T}^{+}, t=t_{k}, k \in \mathbb{N}$, we have from $\left(\mathrm{H}_{8}\right)$ that

$$
\begin{aligned}
x_{i}\left(t_{k}^{+}\right)-x_{i}^{*}\left(t_{k}^{+}\right) & =\left|1-\gamma_{i k}\right|\left|x_{i}\left(t_{k}\right)-x_{i}^{*}\left(t_{k}\right)\right| \\
& \leq\left|x_{i}\left(t_{k}\right)-x_{i}^{*}\left(t_{k}\right)\right|, \quad i=1,2, \ldots, n .
\end{aligned}
$$

Now, let $M>1$ denote an arbitrary real number and set

$$
\left\|\varphi-\varphi^{*}\right\|_{\infty}=\sup _{-\infty<s<0} \max _{1 \leq i \leq n}\left|\varphi_{i}(s)-\varphi_{i}^{*}(s)\right|>0 .
$$

It follows from (50) and Definition 13 that

$$
\begin{array}{r}
V_{i}(t)=\left|y_{i}(t)\right| e_{\varepsilon}(t, a)<M\left\|\varphi-\varphi^{*}\right\|_{\infty}, \\
\forall t \in(-\infty, 0]_{\mathbb{T}}, \quad i=1,2, \ldots, n .
\end{array}
$$

We claim that

$$
\begin{array}{r}
V_{i}(t)=\left|y_{i}(t)\right| e_{\varepsilon}(t, a)<M\left\|\varphi-\varphi^{*}\right\|_{\infty}, \\
\forall t \in(0, \infty)_{\mathbb{T}}, \quad i=1,2, \ldots, n .
\end{array}
$$

If it is not true, in view of the arbitrariness of $M$, there exist $i \in\{1,2, \ldots, n\}$ and $0<t_{i}<t_{1}$ such that

$$
V_{i}\left(t_{i}\right) \geq M\left\|\varphi-\varphi^{*}\right\|_{\infty},
$$

and for $j \neq i, j=1,2, \ldots, n$, we have

$$
V_{j}(t)<M\left\|\varphi-\varphi^{*}\right\|_{\infty}, \quad \forall t \in\left(-\infty, t_{i}\right)_{\mathbb{T}} .
$$

Let $r_{i}=V_{i}\left(t_{i}\right) / M\left\|\varphi-\varphi^{*}\right\|_{\infty}$. Then it follows from (56) and (57) that

$$
\begin{gathered}
r_{i} \geq 1, \quad V_{i}\left(t_{i}\right)=r_{i} M\left\|\varphi-\varphi^{*}\right\|_{\infty}, \\
V_{j}(t)<r_{i} M\left\|\varphi-\varphi^{*}\right\|_{\infty} .
\end{gathered}
$$

Together with (48), (51), (58), and Lemma 10, we obtain

$$
\begin{aligned}
& 0 \leq D^{+}\left|V_{i}^{\Delta}\left(t_{i}\right)\right| \\
&= D^{+}\left|y_{i}^{\Delta}\left(t_{i}\right)\right| e_{\varepsilon}\left(\sigma\left(t_{i}\right), a\right)+\left|y_{i}\left(t_{i}\right)\right| e_{\varepsilon}^{\Delta}\left(t_{i}, a\right) \\
& \leq \epsilon V_{i}\left(t_{i}\right)+\left(1+\epsilon \mu\left(t_{i}\right)\right) \\
& \times\left(\left(-\alpha_{i}^{l} \rho_{i}+\alpha_{i}^{L} B_{i}\right) V_{i}\left(t_{i}\right)+\alpha_{i}^{M} \sum_{j=1}^{n} a_{i j}^{M} f_{j}^{L} V_{j}\left(t_{i}\right)\right. \\
& \quad+\alpha_{i}^{M} \sum_{j=1}^{n} b_{i j}^{M} g_{j}^{L} V_{j}\left(t_{i}-\tau_{i j}\left(t_{i}\right)\right) e_{\varepsilon}\left(t_{i}, t_{i}-\tau_{i j}\left(t_{i}\right)\right) \\
& \quad+\alpha_{i}^{M} \sum_{j=1}^{n} c_{i j}^{M} h_{j}^{L} \\
&\left.\quad \times \int_{0}^{+\infty}\left|K_{i j}(s)\right| e_{\varepsilon}\left(t_{i}, t_{i}-s\right) V_{j}\left(t_{i}-s\right) \Delta s\right) \\
& \leq\left\{\epsilon-\alpha_{i}^{l} \rho_{i}+\left(1+\epsilon \mu\left(t_{i}\right)\right)\left(\alpha_{i}^{L} B_{i}+\alpha_{i}^{M} R_{i}\right)\right\} \\
& \quad \times r_{i} M\left\|\varphi-\varphi^{*}\right\|_{\infty} .
\end{aligned}
$$

Thus,

$$
0 \leq \epsilon-\alpha_{i}^{l} \rho_{i}+\left(1+\epsilon \mu\left(t_{i}\right)\right)\left(\alpha_{i}^{L} B_{i}+\alpha_{i}^{M} R_{i}\right),
$$

which contradicts $\left(\mathrm{H}_{9}\right)$. Hence, (55) holds. It follows that

$$
\begin{array}{r}
\left|x_{i}(t)-x_{i}^{*}(t)\right|<M e_{\ominus \epsilon}\left\|\varphi-\varphi^{*}\right\|_{\infty}, \\
\forall t \in\left(-\infty, t_{1}\right)_{\mathbb{T}}, \quad i=1,2, \ldots, n .
\end{array}
$$


When $t=t_{1}$, the second expression of (48) implies

$$
\begin{aligned}
\left|y_{i}\left(t_{1}^{+}\right)\right| & =\left|y_{i}\left(t_{1}^{-}\right)-\gamma_{i 1} y_{i}\left(t_{1}\right)\right| \\
& \leq\left|1-\gamma_{i 1}\right|\left|y_{i}\left(t_{1}\right)\right| \leq \lim _{t \rightarrow t_{1}^{-}}\left|y_{i}(t)\right| \\
& <M e_{\ominus \epsilon}\left\|\varphi-\varphi^{*}\right\|_{\infty} ;
\end{aligned}
$$

that is,

$$
\left|x_{i}\left(t_{1}^{+}\right)-x_{i}^{*}\left(t_{1}^{+}\right)\right|<M e_{\Theta \epsilon}\left\|\varphi-\varphi^{*}\right\|_{\infty},
$$

where $i=1,2, \ldots, n$. Similar to the step of (51)-(63), we can also prove that

$$
\begin{array}{r}
\left|x_{i}(t)-x_{i}^{*}(t)\right|<M e_{\odot \epsilon}\left\|\varphi-\varphi^{*}\right\|_{\infty}, \\
\forall t \in\left[t_{1}, t_{2}\right)_{\mathbb{T}}, \quad i=1,2, \ldots, n .
\end{array}
$$

When $t=t_{2}$, again, from the second expression of (48), we have

$$
\left|x_{i}\left(t_{2}^{+}\right)-x_{i}^{*}\left(t_{2}^{+}\right)\right|<M e_{\ominus \epsilon}\left\|\varphi-\varphi^{*}\right\|_{\infty}, \quad i=1,2, \ldots, n .
$$

By repeating the same procedure, we obtain

$$
\begin{array}{r}
\left|x_{i}(t)-x_{i}^{*}(t)\right|<M e_{\odot \epsilon}\left\|\varphi-\varphi^{*}\right\|_{\infty}, \\
\forall t \in(0, \infty)_{\mathbb{T}}, \quad i=1,2, \ldots, n .
\end{array}
$$

In view of Definition 13 , the $(\omega / 2)$-antiperiodic solution $x^{*}(t)$ of system (3) is globally exponentially stable. This completes the proof.

\section{Example}

In this section, we give an example to illustrate that our results are feasible.

When $\mathbb{T}=\mathbb{Z}, \sigma(t)=t+1, \mu(t)=1$, we consider the following Cohen-Grossberg neural networks system with impulses:

$$
\begin{aligned}
& x_{i}^{\Delta}(t)=-\alpha_{i}\left(x_{i}(t)\right) \\
& \times\left[\beta_{i}\left(x_{i}(t)\right)-\sum_{j=1}^{n} a_{i j}(t) f_{j}\left(x_{j}(t)\right)\right. \\
&-\sum_{j=1}^{n} b_{i j}(t) g_{j}\left(x_{j}\left(t-\tau_{i j}(t)\right)\right)-\sum_{j=1}^{n} c_{i j}(t) \\
&\left.\times \int_{0}^{+\infty} K_{i j}(s) h_{j}\left(x_{j}(t-s)\right) \Delta s+J_{i}(t)\right], \\
& t \in \mathbb{T}^{+}, \quad t \neq t_{k}, \quad k \in \mathbb{N}, \\
& \Delta x_{i}\left(t_{k}\right)=x_{i}\left(t_{k}^{+}\right)-x_{i}\left(t_{k}^{-}\right)=\frac{1}{800} x_{i}\left(t_{k}\right), \\
& t=t_{k}, \quad i, k=1,2,
\end{aligned}
$$

where

$$
\begin{aligned}
& \alpha_{1}\left(x_{1}(t)\right)=\frac{1}{200}+\frac{1}{300} \cos \left(x_{1}(t)\right) \\
& \alpha_{2}\left(x_{2}(t)\right)=\frac{5}{800}+\frac{1}{800} \cos \left(x_{2}(t)\right) \text {, } \\
& \beta_{1}\left(x_{1}(t)\right)=8 x_{1}(t)+2 \sin \left(x_{1}(t)\right), \\
& \beta_{2}\left(x_{2}(t)\right)=9 x_{2}(t)+4 \sin \left(x_{2}(t)\right) \text {, } \\
& f_{j}(u)=\frac{1}{200} \sin u, \quad g_{j}(u)=\frac{1}{300} \sin u, \\
& h_{j}(u)=\frac{1}{400} \sin u, \quad J_{i}(t)=\sin \left(\frac{\pi}{2} t\right), \\
& K_{i j}(t)=e^{-300 t}, \quad \tau_{i j}(t)=2\left|\cos \left(\frac{\pi}{2} t\right)\right|, \quad i, j=1,2, \\
& \left(a_{i j}(t)\right)_{2 \times 2} \\
& =\left(\begin{array}{cc}
\frac{1}{300}+\frac{1}{200}\left|\sin \left(\frac{\pi}{2} t\right)\right| & \frac{1}{300}-\frac{1}{400}\left|\cos \left(\frac{\pi}{2} t\right)\right| \\
\frac{1}{300}\left|\cos \left(\frac{\pi}{2} t\right)\right| & \frac{1}{400}\left|\sin \left(\frac{\pi}{2} t\right)\right|
\end{array}\right), \\
& \left(b_{i j}(t)\right)_{2 \times 2}=\left(\begin{array}{c|c}
\frac{1}{400}\left|\cos \left(\frac{\pi}{2} t\right)\right| & \frac{1}{500}\left|\sin \left(\frac{\pi}{2} t\right)\right| \\
\frac{1}{600}\left|\sin \left(\frac{\pi}{2} t\right)\right| & \frac{1}{700}\left|\sin \left(\frac{\pi}{2} t\right)\right|
\end{array}\right),
\end{aligned}
$$

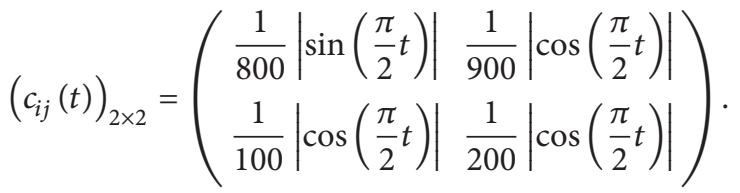

By calculation, we have

$$
\begin{gathered}
\alpha_{1}^{M}=\frac{5}{600}, \quad \alpha_{1}^{m}=\alpha_{1}^{l}=\frac{1}{600}, \quad \alpha_{2}^{M}=\frac{3}{400}, \\
\alpha_{2}^{m}=\alpha_{2}^{l}=\frac{1}{200}, \quad \alpha_{1}^{L}=\frac{1}{300}, \quad \alpha_{2}^{L}=\frac{1}{800}, \\
\rho_{1}=6, \quad \delta_{1}=10, \quad \rho_{2}=5, \quad \delta_{2}=13, \\
f_{j}^{M}=f_{j}^{L}=\frac{1}{200}, \quad g_{j}^{M}=g_{j}^{L}=\frac{1}{300}, \\
h_{j}^{M}=h_{j}^{L}=\frac{1}{400}, \quad J_{i}^{M}=1, \\
i=\frac{4}{800}, \\
\left(a_{i j}^{M}\right)_{2 \times 2}=\left(\begin{array}{c}
\frac{5}{600} \frac{1}{300} \\
\frac{1}{300} \frac{1}{400}
\end{array}\right)
\end{gathered}
$$




$$
\begin{aligned}
\left(b_{i j}^{M}\right)_{2 \times 2} & =\left(\begin{array}{cc}
\frac{1}{400} & \frac{1}{500} \\
\frac{1}{600} & \frac{1}{700}
\end{array}\right), \\
\left(c_{i j}^{M}\right)_{2 \times 2} & =\left(\begin{array}{cc}
\frac{1}{800} & \frac{1}{900} \\
\frac{1}{100} & \frac{1}{200}
\end{array}\right) .
\end{aligned}
$$

Therefore, $E_{1}=0.02407, E_{2}=0.05825$. Take $\epsilon=10^{-6}$ and $\eta=10^{-3}$, and then

$$
l_{1} \approx-0.009998, \quad l_{2} \approx-0.024999 .
$$

It is easy to see that $\left(\mathrm{H}_{1}\right)-\left(\mathrm{H}_{9}\right)$ hold. According to Theorems 16 and 17 , system (67) has a 2-antiperiodic solution which is globally exponentially stable.

\section{Conflict of Interests}

The authors declare that there is no conflict of interests regarding the publication of this paper.

\section{Acknowledgments}

The authors are very grateful to the reviewers and the editor for their valuable suggestions and comments. This work was supported by National Natural Science Foundation of China (11271261) and FP7-PEOPLE-2012-IRSES-316338.

\section{References}

[1] M. A. Cohen and S. Grossberg, "Absolute stability of global pattern formation and parallel memory storage by competitive neural networks," IEEE Transactions on Systems, Man, and Cybernetics, vol. 13, no. 5, pp. 815-826, 1983.

[2] Y.-K. Li, "Existence and stability of periodic solutions for Cohen-Grossberg neural networks with multiple delays," Chaos, Solitons and Fractals, vol. 20, no. 3, pp. 459-466, 2004.

[3] J. Liu, "Global exponential stability of Cohen-Grossberg neural networks with time-varying delays," Chaos, Solitons and Fractals, vol. 26, no. 3, pp. 935-945, 2005.

[4] H.-J. Jiang, J.-D. Cao, and Z.-D. Teng, "Dynamics of CohenGrossberg neural networks with time-varying delays," Physics Letters A: General, Atomic and Solid State Physics, vol. 354, no. 5-6, pp. 414-422, 2006.

[5] Z. Li and K. Li, "Stability analysis of impulsive Cohen-Grossberg neural networks with distributed delays and reaction-diffusion terms," Applied Mathematical Modelling, vol. 33, no. 3, pp. 13371348, 2009.

[6] Y. Li, X. Chen, and L. Zhao, "Stability and existence of periodic solutions to delayed Cohen-Grossberg BAM neural networks with impulses on time scales," Neurocomputing, vol. 72, no. 7-9, pp. 1621-1630, 2009.

[7] W. Su and Y. Chen, "Global robust stability criteria of stochastic Cohen-Grossberg neural networks with discrete and distributed time-varying delays," Communications in Nonlinear Science and Numerical Simulation, vol. 14, no. 2, pp. 520-528, 2009.
[8] X. Liao, J. Yang, and S. Guo, "Exponential stability of CohenGrossberg neural networks with delays," Communications in Nonlinear Science and Numerical Simulation, vol. 13, no. 9, pp. 1767-1775, 2008.

[9] W. Zhao, "Global exponential stability analysis of CohenGrossberg neural network with delays," Communications in Nonlinear Science and Numerical Simulation, vol. 13, no. 5, pp. 847-856, 2008.

[10] B. W. Liu, "New convergence behavior of solutions to CohenGrossberg neural networks with delays and time-varying coefficients," Physics Letters A, vol. 372, no. 2, pp. 117-123, 2008.

[11] W.-R. Zhao, "Dynamics of Cohen-Grossberg neural network with variable coefficients and time-varying delays," Nonlinear Analysis: Real World Applications, vol. 9, no. 3, pp. 1024-1037, 2008.

[12] Y.-H. Xia, Z.-K. Huang, and M.-A. Han, "Exponential pstability of delayed Cohen-Grossberg-type BAM neural networks with impulses," Chaos, Solitons \& Fractals, vol. 38, no. 3, pp. 806-818, 2008.

[13] C. Bai, "Global exponential stability and existence of periodic solution of Cohen-Grossberg type neural networks with delays and impulses," Nonlinear Analysis: Real World Applications, vol. 9, no. 3, pp. 747-761, 2008.

[14] Q. Song and J. Zhang, "Global exponential stability of impulsive Cohen-Grossberg neural network with time-varying delays," Nonlinear Analysis: Real World Applications, vol. 9, no. 2, pp. 500-510, 2008.

[15] W. Wu, B. T. Cui, and X. Y. Lou, "Global exponential stability of Cohen-Grossberg neural networks with distributed delays," Mathematical and Computer Modelling, vol. 47, no. 9-10, pp. 868-873, 2008.

[16] K. Li, "Stability analysis for impulsive Cohen-Grossberg neural networks with time-varying delays and distributed delays," Nonlinear Analysis. Real World Applications, vol. 10, no. 5, pp. 2784-2798, 2009.

[17] H.-J. Jiang, J.-D. Cao, and Z.-D. Teng, "Anti-periodic solutions for Cohen-Grossberg neural networks with bounded and unbounded delays," Physics Letters A: General, Atomic and Solid State Physics, vol. 14, no. 7, pp. 3134-3140, 2009.

[18] Y.-K. Li and J.-Y. Shu, "Anti-periodic solutions to impulsive shunting inhibitory cellular neural networks with distributed delays on time scales," Communications in Nonlinear Science and Numerical Simulation, vol. 16, no. 8, pp. 3326-3336, 2011.

[19] C. Ou, "Anti-periodic solutions for high-order Hopfield neural networks," Computers \& Mathematics with Applications, vol. 56, no. 7, pp. 1838-1844, 2008.

[20] Y. Chen, J.-J. Nieto, and D. O. O’Regan, “Anti-periodic solutions for fully nonlinear first-order differential equations," Mathematical and Computer Modelling, vol. 46, no. 9-10, pp. 1183-1190, 2007.

[21] L. Peng and W. Wang, "Anti-periodic solutions for shunting inhibitory cellular neural networks with time-varying delays in leakage terms," Neurocomputing, vol. 111, pp. 27-33, 2013.

[22] J. Shao, "Anti-periodic solutions for shunting inhibitory cellular neural networks with time-varying delays," Physics Letters A, vol. 372, no. 30, pp. 5011-5016, 2008.

[23] J. Gao, Q. Wang, and L.-W. Zhang, "Existence and stability of almost-periodic solutions for cellular neural networks with time-varying delays in leakage terms on time scales," Applied Mathematics and Computation, vol. 237, pp. 639-649, 2014. 
[24] M. Bohner and A. Peterson, Dynamic Equations on Time Scales: An Introduction with Applications, Birkhauser, Boston, Mass, USA, 2001.

[25] Z.-Q. Zhang and K.-Y. Liu, "Existence and global exponential stability of a periodic solution to interval general bidirectional associative memory (BAM) neural networks with multiple delays on time scales," Neural Networks, vol. 24, no. 5, pp. 427439, 2011.

[26] Y.-Q. Wang, Q.-W. Tu, Q. Wang, and C. Hu, "Positive periodic solutions of impulsive functional differential equations on time scales with a parameter," International Journal of Dynamical Systems and Differential Equations, vol. 3, no. 1-2, pp. 12-23, 2011.

[27] R. Agarwal, M. Bohner, and A. Peterson, "Inequalities on time scales: a survey," Mathematical Inequalities and Applications, vol. 4, no. 4, pp. 535-557, 2001.

[28] A. Ardjouni and A. Djoudi, "Existence of periodic solutions for nonlinear neutral dynamic equations with variable delay on a time scale," Communications in Nonlinear Science and Numerical Simulation, vol. 17, no. 7, pp. 3061-3069, 2012.

[29] D. O'regan, Y.-J. Chu, and Y.-Q. Chen, Topological Degree Theory and Application, Taylor \& Francis, New York, NY, USA, 2006.

[30] G. S. Guseinov, "Integration on time scales," Journal of Mathematical Analysis and Applications, vol. 285, no. 1, pp. 107-127, 2003. 


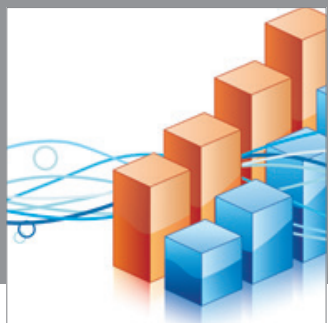

Advances in

Operations Research

mansans

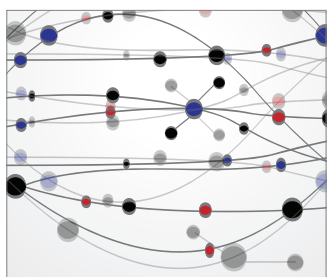

The Scientific World Journal
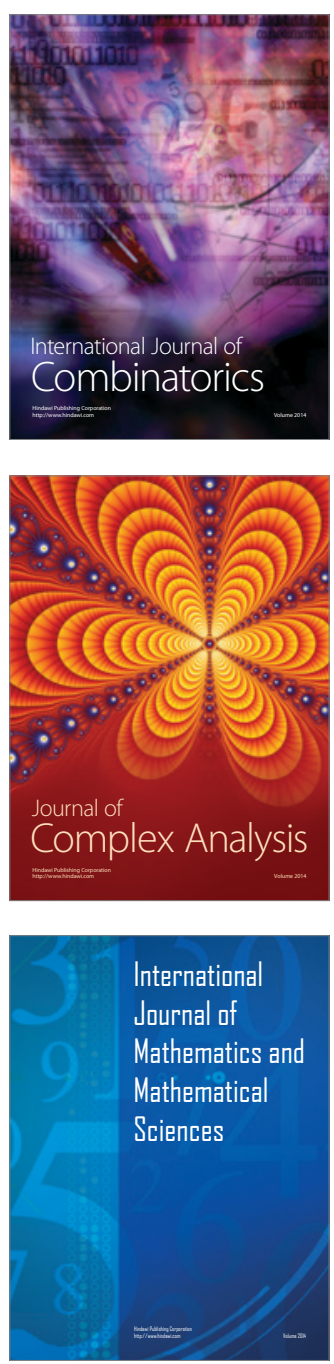
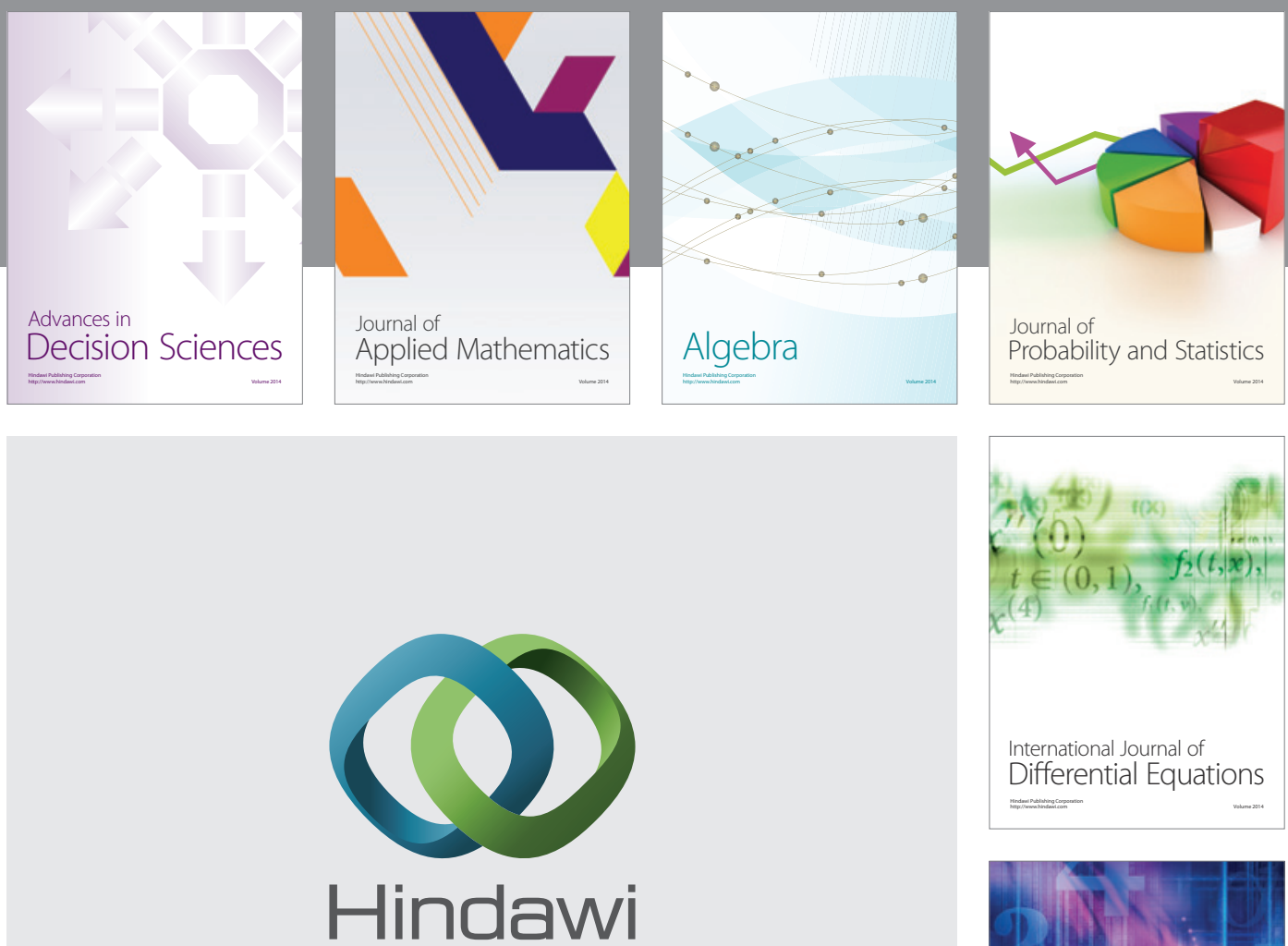

Submit your manuscripts at http://www.hindawi.com
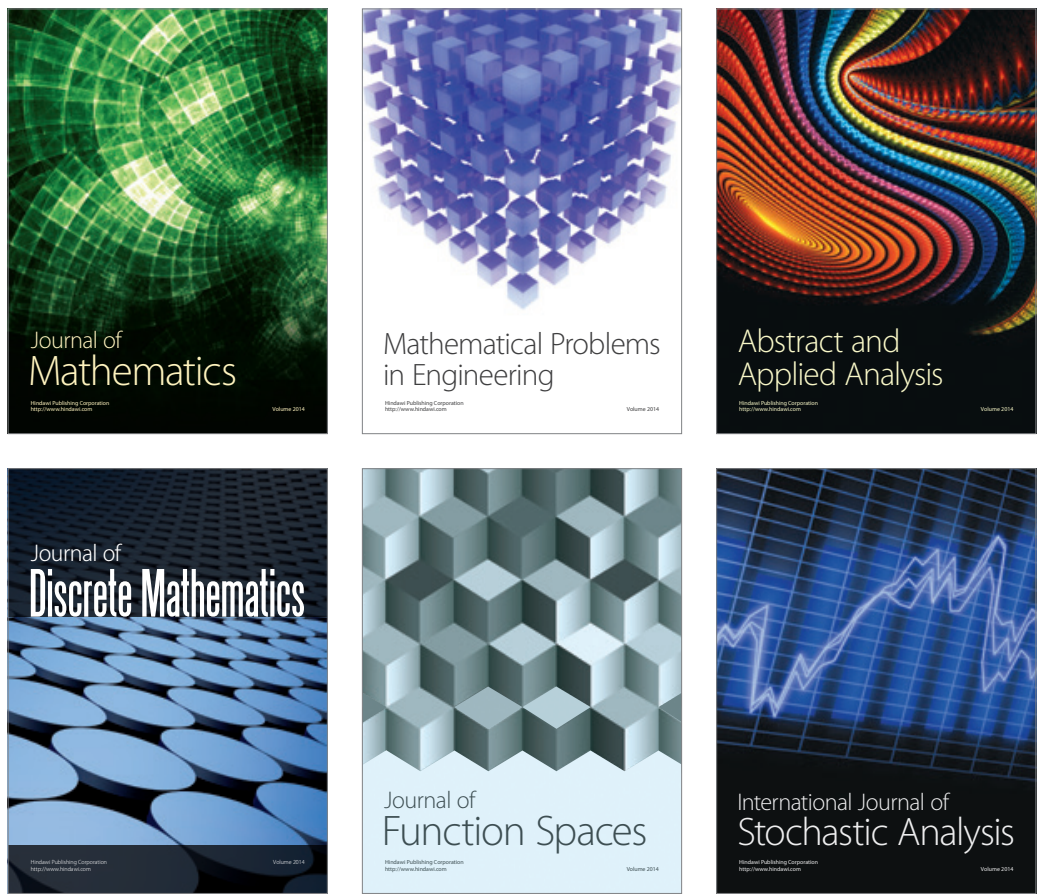

Journal of

Function Spaces

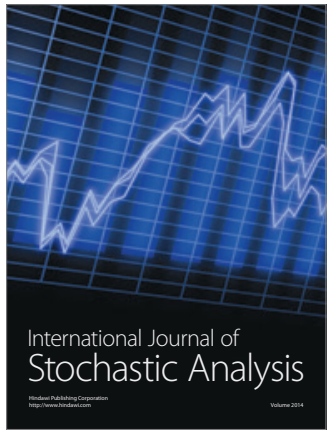

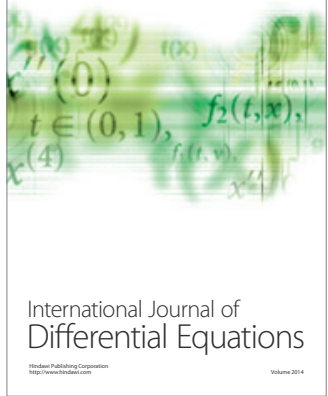
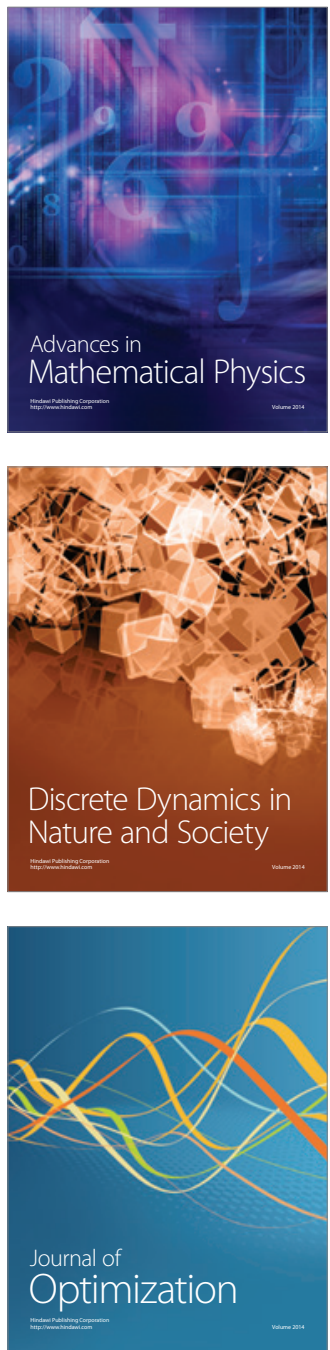